\title{
Die Schweizerische Gesellschaft für Medizinische Informatik SGMI
}

\author{
Behandlung und Pflege von Patientinnen und Patienten sind ohne professio-
} nell geplante Erhebung und Verarbeitung von Informationen nicht möglich. Die Informationslogistik ist wesentlich für die Qualität der interdisziplinären Patientenversorgung, sowohl in Gesundheitsnetzwerken als auch für Spitäler. Die Aufarbeitung und Darstellung relevanter Daten ist Voraussetzung für diagnostische und therapeutische Entscheidungen. Die Schweizerische Gesellschaft für Medizinische Informatik SGMI befasst sich mit all diesen Facetten der Informations- und Kommunikationstechnologie (ICT) im Gesundheitswesen.

Eusebio Passaretti ${ }^{a}$,

Benno Sauter ${ }^{b}$, Martin Denz ${ }^{c}$, Christian Lovis ${ }^{d}$

a Universitätsspital Basel, Präsident SGMI

b Dr. med., Gesundheitszentrum Fricktal, Vorstandsmitglied SGMI

c Dr. med., ETH Lausanne, Vorstandsmitglied SGMI

d Prof. Dr. med., Universitätsspital Genf, Vizepräsident SGMI
Korrespondenz:

Schweizerische Gesellschaft

für Medizinische Informatik SGMI Im Lehn

CH-3116 Kirchdorf

pia.jäggi@sgmi.ch

www.sgmi-ssim.ch
Die SGMI besteht seit über 20 Jahren und ist der interdisziplinäre Verband aller Fachpersonen, die sich im Berufsalltag mit ICT im Gesundheitswesen auseinandersetzen. Die etwa 300 Mitglieder stammen aus allen Berufsgruppen, arbeiten auf allen Hierarchiestufen, in staatlichen und nichtstaatlichen Organisationen, grossen und kleinen Spitälern, sind in Arztpraxen oder Krankenkassen tätig, kommen aber auch aus privatwirtschaftlichen Unternehmungen im Gesundheitswesen. Zahlreiche Mitglieder der SGMI sind auf verschiedenen Gebieten der ICT im Gesundheitswesen als national und international anerkannte Experten und Wissenschaftler tätig. Als nationale Gesellschaft pflegt die SGMI Beziehungen sowohl zu medizinischen als auch zu technischen Fach- und Berufsorganisationen. Zudem ist die SGMI die nationale Repräsentantin der International Medical Informatics Association (IMIA) und der European Federation for Medical Informatics (EFMI). Die Experten der SGMI leisten durch ihre Mitarbeit in nationalen und internationalen Gremien einen wichtigen Beitrag zur Weiterentwicklung des Gesundheitswesens.

Die SGMI widmet sich folgenden Themen:

\section{Gesundheitsmanagement, klinisches und Logistikmanagement}

Die Verbesserung der Patientensicherheit, die Verbesserung von Qualität und Effizienz sowie die Kostenreduktion im Gesundheitswesen haben auch international hohe Priorität. Dafür benötigen wir erschwingliche und dennoch qualitativ hochstehende Informationssysteme, die messbare Prozessverbesserungen ermöglichen und zur Optimierung der Ressourcenallokation beitragen. Als Management-, Forschungs- und anonyme Meldesysteme tragen sie z. B. dazu bei, unerwünschte Medikamentennebenwirkungen und lebensbedrohliche Zwischenfälle $\mathrm{zu}$ verhindern. Der alleinige Technologieeinsatz reicht jedoch nicht aus: Zum Erfolg führt nur die Ausrichtung am gesamten Organisationssystem in Zusammenarbeit mit den darin tätigen Menschen.

\section{Elektronische Patientenakte und Gesundheitsinformationssysteme} Voraussetzung für die obenerwähnten Lösungsansätze sind elektronisch nutzbare Patientendaten, die idealerweise nach bestehenden Standards strukturiert und in einer Datenbank verfügbar sind. Auf internationaler Ebene stehen Länder wie beispielsweise Frankreich und England unmittelbar davor, ihren Bürgerinnen und Bürgern eine lebenslange, patienteneigene elektronische Akte bereitzustellen. Die SGMI ist international an diesen Entwicklungen mitbeteiligt und fördert mit ihrem Fachwissen derzeit auch kantonale Projekte mit derselben Stossrichtung.

\section{Entscheidungsunterstützung, Informationsmanagement und Wissens- aufbereitung}

Wir werden sowohl im Internet als auch bezüglich medizinischer Publikationen von einer Informationsflut überrollt. Das Management dieser Informationen und deren sinnvolle Nutzung sind entscheidend. Die SGMI beteiligt sich seit 
Jahren an der Diskussion und der Entwicklung von Systemen, welche dazu beitragen, das richtige Wissen zur richtigen Zeit am richtigen Ort zur Verfügung zu stellen. Auch der «informierte Patient» profitiert von den Entwicklungen der SGMI: Sie steht hinter der Gründung von Healthon-the-Net (www.hon.ch), einer Stiftung, die dabei hilft, vertrauenswürdige medizinische und Gesundheitsinformationen im Internet zu finden.

Weitere Themenbereiche, zu denen die SGMI massgebliche Beiträge leistet, sind die: Bioinformatik, Public Health Informatics, medizinische Signal- und biomedizinische Bildverarbeitung.
Ob über medizinische Informatik, E-Health, Telemedizin oder ICT im Gesundheitswesen debattiert wird: die SGMI hat die dafür notwendigen Grundlagen seit mehr als 20 Jahren erarbeitet, vieles davon umgesetzt und verfügt über wertvolle Erfahrung. In der Diskussion um die künftigen Entwicklungen des Schweizer Gesundheitswesens wird die SGMI weiterhin auch die Standardisierung und Prozessoptimierung mittels ICT fördern. Dafür setzen wir unser nationales und internationales Netzwerk als Wissens- und Expertenpool ein. Werden auch Sie ein Teil davon! 